\title{
La comunicación en redes sociales: percepciones y usos de las Ong españolas
}

\author{
Social Network Communication: \\ Perceptions and Uses for Spanish NGOs
}

\author{
(ARTÍCULO)
}

ISIDORO ARROYO ALMARAZ, Universidad Rey Juan Carlos, Madrid, España. (isidoro.arroyodurjc.es) ANTONIO J. BALADRÓN PAZOS, Universidad Rey Juan Carlos, Madrid, España.(antonio.baladronQurjc.es) REBECA MARTíN NIETO, Universidad Rey Juan Carlos, Madrid, España. (rebeca.martinQurjc.es)

- Recibido: 27/marzo/2013. Aceptado: 28/mayo/2013

\section{RESUMEN}

El objetivo de esta investigación es conocer cuáles son las percepciones y usos que las ONG españolas analizadas hacen de las redes sociales. Se realiza una investigación cuantitativa con cuestionarios y otra cualitativa con dos grupos focales para valorar los aspectos positivos y negativos que afectan al uso de las redes sociales. Se analiza el papel de estas en la planificación de la comunicación y se adelantan expectativas de futuro de mejora de la creatividad e innovación de los mensajes y de perfeccionamiento en la medición de eficacia de la comunicación en las redes sociales.

DOI: $10.7764 /$ cdi.32.497

Palabras clave: comunicación, social, ONG, redes sociales, eficacia, creatividad

\section{ABSTRACT}

The aim of the current investigation is to discover the perceptions and uses of Social Networks for Spanish NGOs. Two research lines have been stated, a quantitative one based on a poll, and another one qualitative and based on the pro/con valuation of two designed groups. The role of Social Networks in the communication plan of the NGOs has been analyzed, foreseeing the possible upcoming needs. The scope of the reference analysis includes aspects of creativity and innovation in the message release, as well as the measure methodologies optimization within the Social Networking.

Keywords: communication, social, NGO, social network, efficiency, creativity

1. Financiamiento: Ministerio de Ciencia y Tecnología, Programa Nacional de Proyectos de Investigación Fundamental no Orientada 2008-2011. Proyecto I+D+I «Desarrollo de un modelo de eficacia de la comunicación persuasiva del tercer sector en las redes sociales» (CS02009-11203). 
La comunicación social es el instrumento que las ONG utilizan, cada vez más, para dar a conocer sus principios y acciones. Al principio se entendía como un aspecto secundario, sobre el que primaban los proyectos que desarrollaban en los países beneficiarios. Sin embargo, la comunicación está tomando relevancia para estas organizaciones puesto que "los medios en sí no hacen la movilización social en el sentido estricto, pero facilitan el trabajo de las ONG para la movilización de conciencias y voluntades, fomentando poderosos movimientos en la opinión pública" (Grzybowski, 2001, p. 31). Por ende, es la comunicación la que posibilita la creación y mantenimiento de vínculos sólidos entre las ONG y sus socios, colaboradores y públicos, tal y como han expresado diversos autores (Herranz de la Casa, 2009; Cerdá, 2003; Erro, 2002; Aranguren, 1998; Angulo, 1998; Aguadero, 1993).

Hasta hace unos años, los medios de comunicación convencionales eran para las ONG los altavoces más eficaces de sus mensajes. Pero de la abundancia de organizaciones y la dificultad para aparecer en esos medios ha surgido la conveniencia de buscar otros canales, cobrando relevancia primero la comunicación web (Martín, 2010) y poco después las redes sociales por el abaratamiento que suponen para los procesos comunicativos de las ONG (Sampedro, 2006, p. 2)

La situación de crisis económica vivida en España en los últimos años está potenciando el uso de estos nuevos canales, ya que su costo es considerablemente inferior a lo que podrían suponer las grandes campañas publicitarias de carácter masivo en los medios tradicionales. Es una tendencia que las ONG comparten con el resto de actores publicitarios del panorama empresarial e institucional, obligados a reducir drásticamente sus presupuestos publicitarios. Los nuevos medios, en especial las redes sociales, hacen posible una comunicación directa con sus públicos sin necesidad de realizar costosas inversiones en compra de espacios y tiempos publicitarios. Esta situación ha hecho cambiar de forma importante el uso que las ONG están haciendo de las redes sociales y que se apuntaba como tendencia más que como una realidad palpable en el momento de la realización del estudio aquí presentado, centrado en la primera fase de la crisis en la que aún no se preveía el fuerte azote que esta llevaría a cabo sobre el tejido social y empresarial español.

Además, como señala Orihuela, los nuevos medios aúnan tres dimensiones: "Comunicación, al permitir poner en común los conocimientos; comunidad, al ayudar a integrar comunidades; y cooperación, al ayudar a hacer cosas juntos" (Ramil, 2005). Un ejemplo de esto último son las acciones de cibervoluntariado; se utilizan las redes sociales para comunicar los logros de la organización, solicitar la implicación de los cibervoluntarios y comunicarles nuevas necesidades de acción, así como para tareas de sensibilización, alcanzando el compromiso activo de los miembros de la red social. En este sentido, podemos definir las redes sociales como un aparato social con potencia revolucionaria y emancipadora, en tanto puede incluir a todos, y en tanto debe incluir a todos. Por lo cual se utilizan para construir comunidades virtuales donde sus usuarios interactúan con personas de todo el mundo buscando, como objetivo último, la inclusión social. (Zafra, 2010, p. 73). Para alcanzar estos objetivos, "la mayoría de las ONG creen que Twitter, Facebook y YouTube son los medios sociales idóneos. Cada medio contribuye a fines distintos y la suma de todos ellos genera la nueva forma de comunicar. Pero en todos ellos es esencial, en primer lugar, la presencia del video viral para ser compartido y comentado en las diferentes redes sociales y, en segundo lugar, cómo lo difunde el usuario según se encuentre en una u otra red social" (Arroyo, Baños y Van Wyk, 2013, p. 331). Por lo tanto, YouTube es considerado en esta investigación como instrumento fundamental para buscar un efecto de viralidad a través de las llamadas técnicas de comunicación mediante rumor digital

En este sentido, este nuevo medio presenta para las ONG la potencialidad ya probada en otros movimientos sociales para "saltar de la red a la calle, al espacio público y a los medios masivos" (Ferreras, 2011, s.p.) como sucedió recientemente con el movimiento 15-M en España. Hace unos años, Marí Saéz concluía, en un estudio sobre el uso de internet por parte del tercer sector, que "se infrautiliza el potencial del trabajo en red y de construcción colectiva del conocimiento" (2001, p. 462), función que sin duda se debería ver reforzada con el recurso a las redes sociales.

Pese a las posibilidades que abren las redes para las ONG este ámbito apenas ha sido analizado científicamente, siendo un objeto de estudio con una proyección importante y sobre el cual es necesario desarrollar investigaciones experimentales que sean referentes en el ámbito. Sí se han llevado a cabo diversos trabajos sobre la comunicación persuasiva de las ONG -alguno de especial valor por su rigor y su avance en el conocimiento (Martín, 2010; Sabre, 2011; Arroyo y Martín, 2011) - e incluso sobre su aplicación a otros espacios concretos de la comunicación 2.0 como son los blogs (Herranz de la Casa y Cabezuelo Lorenzo, 2009; Berrios, 2005), pero no específicamente sobre redes sociales, salvo alguno que otro estudio no experimental (Baraybar, 2009). Fuera de España, con un objetivo y metodología similar a la del estudio que aquí 
presentamos, cabe señalar una reciente investigación sobre adopción y uso de las TIC por parte de las ONG de Nueva Zelanda (Zorn, Flanagin y Shoham, 2011), algunas de cuyas conclusiones guardan similitudes con las obtenidas en nuestra investigación. Por último, cabría mencionar alguna encuesta llevada a cabo por entidades independientes como Citizen Schools y Public Learning Media Lab (2008) sobre el uso de la web social por parte de las organizaciones.

\section{METODOLOGÍA}

El objetivo de esta investigación es conocer cuáles son las percepciones y usos de las redes sociales como herramienta de comunicación por parte de las ONG con sede en España en el contexto de los primeros años de la crisis económica que afecta a ese país desde 2008, cuando la mayoría de las ONG recurrían a la financiación pública. ${ }^{2}$ Los objetivos específicos, principalmente tres: identificar las motivaciones y frenos que las ONG detectan a la hora de usar las redes como canal de comunicación; observar las potencialidades que encuentran en su uso, y conocer las estrategias comunicativas que plantean estas organizaciones de cara al desarrollo de la comunicación presente y futura a través de redes sociales.

Para la consecución de los objetivos se combinó un método de investigación cuantitativo y otro cualitativo, implementados en 2010 y 2011; en concreto, un cuestionario y un grupo de discusión, ${ }^{3}$ contándose en ambos con la participación de gestores de la comunicación de las ONG. Se optó por una primera fase de investigación de naturaleza cuantitativa, apoyada por una segunda fase basada en grupos de discusión con el fin de evaluar cualitativamente los datos arrojados por los cuestionarios y profundizar en las relaciones sugeridas por el análisis cuantitativo. Por tanto, se siguió la estrategia de la triangulación a la que se refiere Bericat (1998), ya que el objeto de estudio y los objetivos son los mismos para los dos métodos de investigación utilizados, permitiéndose así reforzar la validez de los resultados.

La elección del cuestionario como instrumento de la primera fase se debió, principalmente, a que proporciona una gran riqueza en información cuantitativa, algo fundamental para nuestro objeto de estudio por la casi inexistencia de investigaciones científicas sobre el mismo. Se optó por un cuestionario estandarizado, programado o estructurado, basado en la utilización de las mismas preguntas: unas generales y abiertas que inciden en las potencialidades del medio, como por ejemplo: ¿Qué barreras o miedos encuentras a la hora de lanzarte a las redes sociales? y otras específicas y de respuesta concreta, como por ejemplo ¿Mides la incidencia de tus mensajes en las redes sociales? que inciden en el uso real que hacen de esas potencialidades. Las preguntas se presentaron en el mismo orden para todos los participantes. Para el tratamiento de los datos recogidos en los cuestionarios se utilizó el análisis de contenido, porque es una técnica que permite sistematizar y objetivar las características de los mensajes. Esta técnica permitió considerar cada una de las respuestas a cada una de las preguntas del cuestionario cuyo contenido podría ser objetivado y a partir de ellas formular inferencias reproducibles y válidas.

Respecto a la segunda fase de la investigación, la selección del grupo de discusión estuvo motivada por su capacidad para generar material cualitativo a partir de la interacción grupal, ya que los focus group permiten "la interdependencia de sus miembros y el hecho de que cada integrante actúa en relación con sus propias necesidades, manifestando su vivencia, su experiencia y su punto de vista" (Soler, 1999, p. 80). De este modo, se consigue información a la que no se podría llegar en las entrevistas individuales u otras técnicas cualitativas. Esto era fundamental para los objetivos planteados en nuestro estudio, ya que se trataba de obtener información sobre los usos y percepciones de las redes sociales por parte de las ONG yendo más allá de las opiniones individuales y obteniendo información agregada que pudiera servir para una mejor comprensión de los resultados de la primera fase. En términos generales, esa información sobre usos y percepciones se consideró que debiera ser investigada a través de los testimonios de las personas responsables de los departamentos de comunicación de las ONG antes que mediante el análisis directo de sus perfiles en las redes, por la gran disparidad de tamaño, presupuesto y características de las organizaciones participantes en la investigación y el desigual uso real que existía entre unas y otras.

\section{SELECCIÓN DE LA MUESTRA}

Para seleccionar la muestra se empezó trabajando con las 41 ONG calificadas para el desarrollo por la Agencia Española de Cooperación Internacional ${ }^{4}$ (ONGD). Esta calificación supone que todas ellas han pasado un proceso de reconocimiento a su gestión. Dada la cantidad, calidad, rigor y concreción de los datos que las ONGD facilitan a la Aecid durante el proceso, se considera que el hecho de estar calificadas es suficientemente significativo como para centrar en ellas esta investigación. Trece de ellas, que se corresponden con el 31,7\% de las ONGD calificadas, finalmente participaron en la presente investigación. Para completar la muestra se contactó con más de veinte organizaciones más que llevaban cuatro años realizando comunicación
2. Hasta 2010, nueve de cada diez $O N G$ recurrían a la financiación pública, según El Anuario del Tercer Sector de Acción Social en España 2012 (Fundación Luis Vives, 2012).

3. Los focus group o grupos de discusión se celebraron los días 22 de marzo y 15 de abril de 2010 en las instalaciones de la Facultad de Ciencias de la Comunicación de la Universidad Rey Juan Carlos. Las preguntas de los cuestionarios fueron formuladas en entrevistas personales cara a cara programadas con los diferentes responsables de comunicación de las organizaciones durante el último trimestre del año 2009. Los cuestionarios fueron cumplimentados en el transcurso de esas entrevistas.

4. En los años 2005, 2007 y 2009. 
5. Siguiendo el orden de recepción de los cuestionarios, estas son: Anesvad,

Farmacéuticos Mundi, Salud para Todos, Pacto Mundial, FAD, Codespa, Movimiento por la Paz, Adaner, 5 al Día, Cáritas Getafe, Greenpeace, Setem, Un Solo Mundo, Asamblea Cooperación por la Paz, Cruz Roja, Juntos para Jugar, Maison des Enfants, Fundación Vicente Ferrer, Fundación Nelson Mandela, Comercio Justo Web, Acsur las Segovias, Acción contra el Hambre, Manos Unidas, Fundación Tomillo, Cáritas Española, Jóvenes del Tercer Mundo, Abenin, Fundación San Ezequiel, Cesal, Asociación Miguel Fenollera, IntermónOxfam, Asza y Accem.

6. Destacan las siguientes ONG: Adaner, Asamblea de Cooperación por la Paz, Fad Greenpeace, Cáritas, Cruz Roja Madrid, Movimiento por la Paz, Salud para Todos, Setem y 5 al día. social por medios convencionales y no convencionales. La iniciativa tuvo una excelente acogida, siendo finalmente 35 organizaciones las que entregaron el cuestionario cumplimentado.

Para seleccionar la muestra de los grupos de discusión se efectuaron dos convocatorias entre estas 35 organizaciones y se constituyeron dos grupos (GD1 y GD2). Habitualmente, se recomienda la organización de dos grupos de discusión por cada categoría relevante para el estudio, con el fin de alcanzar un mínimo nivel de saturación. Como corresponde a una técnica cualitativa, como es el grupo de discusión, el objetivo no era conseguir representatividad estadística, sino obtener discursos pertinentes y relevantes de acuerdo con los objetivos de la investigación. Al no utilizarse el método como autosuficiente para el logro de los objetivos propuestos, sino complementario, y precisamente por ser innecesaria una categorización de los participantes de acuerdo a esos objetivos, el número final de grupos se limitó a dos. En este sentido, Gaitán y Piñuel (1998) señalan que un número reducido de grupos evita la tendencia a la redundancia o ruido, de ahí que establezca en dos el número mínimo necesario para asegurar la contrastabilidad de los datos. Finalmente, participaron 16 miembros de ONG con sede en España, todos vinculados a los departamentos responsables de la comunicación de las mismas. ${ }^{6}$

Respecto a los grupos de discusión, a partir de la información registrada se elaboraron sendos informes de acuerdo al que Krueger denomina modelo interpretativo y en el que, como indica Valles, "sobre la base de la reducción descriptiva de la información [...] se añade un esfuerzo analítico e interpretativo de mayor calado" (2000, p. 328). En cuanto a los cuestionarios, a partir del análisis de contenido se realizaron inferencias que se organizaron en dos bloques: uno referido a las percepciones que los responsables de comunicación tienen sobre las redes sociales, tanto en su aspecto de posibilidades como en el de miedos; y otro, a los usos reales que hacen de dichas redes, tanto en la delimitación del tipo de presencia que tienen como en las expectativas de futuras mejoras.

\section{RESULTADOS}

\section{DE LA FASE CUANTITATIVA}

A continuación se presentan los datos cuantitativos de frecuencia de aparición de las respuestas de los cuestionarios, indistintamente de en qué pregunta aparecieran. Son, por tanto, el número de coincidencias de una afirmación que inciden en un concepto. Las tablas recogen conceptos agrupados por significados similares, entre los que se destacan en negrita los más frecuentes; estos a su vez están ordenados de mayor a menor frecuencia.

\section{Percepciones sobre las redes sociales}

Comenzando por las percepciones que las ONG tienen de las redes sociales, en primer lugar en lo referente a las posibilidades de las redes (Tabla 1), los valores percibidos como más importantes por las 35 ONG participantes son: la proximidad, la difusión de mensajes, la rapidez o la gratuidad. La proximidad, con una frecuencia de 24 coincidencias de respuesta, es entendida principalmente como la posibilidad de establecer un contacto directo con los públicos y de tener con ellos una actitud activa; igualmente se considera la capacidad de difusión de mensajes (con 24 coincidencias). Por otra parte, destaca la rapidez (18) con la que se puede compartir la información con los públicos gracias a la inmediatez (7) entre el tiempo en que se produce la información y el tiempo en que se actualiza la misma dentro de la red social, satisfaciendo la demanda de información (8).

En segundo lugar, entre los frenos que las ONG perciben para utilizar las redes sociales (Tabla 2) destaca, por encima de cualquier otro, el miedo a la falta de control de la comunicación (con 27 coincidencias), especialmente en lo tocante al uso incorrecto que se pudiera hacer de los datos e informaciones que las organizaciones proporcionan (27). En segundo lugar merece la pena mencionar el miedo a las prácticas que pudieran vulnerar derechos (22)

\section{Usos de las redes sociales}

En cuanto al uso que las ONG hacen de las redes, en la Tabla 3 se puede observar que más de la mitad de la muestra manifiesta que tiene presencia en redes sociales y de estas, 17 lo hacen en Facebook y 11 en YouTube. En menor medida están en Twitter o Tuenti y muy pocas en Flickr. Estos datos están en consonancia con los de las ONG norteamericanas publicados en EE.UU. (Quinn y Berry, 2010), que indican que principalmente manifiestan tener presencia en Facebook (73\%), Twitter (56\%), blogs (45\%) o plataformas para compartir videos (49\%) así como con los recogidos en la encuesta llevada a cabo por Citizen Schools y Public Learning Media Lab (2008), según la cual los blogs, Facebook y YouTube también son los canales de la web social más utilizados por las organizaciones. Las pequeñas diferencias vienen determinadas por el auge de Twitter posterior a la toma de datos de esta investigación y el papel dominante que tienen los blogs en la web 2.0.

Por otra parte, las 20 ONG que están presentes en redes sociales las consideran medios eficaces, no una moda pasajera; sin embargo, el $65 \%$ percibe que no se aprovechan todas las ventajas que ofrecen y solo el 10\% considera que sí. Asimismo, el 
Tabla 1. Respuestas objetivadas del cuestionario sobre posibilidades de las redes sociales

\begin{tabular}{|c|c|}
\hline Concepto & Frecuencia \\
\hline Proximidad & 24 \\
\hline Contacto directo y actitud activa & 20 \\
\hline Temas de interés para PO & 7 \\
\hline Lenguaje y comunicación novedoso y actual & 1 \\
\hline Difusión & 24 \\
\hline Llegar al gran público (a la masa) & 15 \\
\hline Movilización y multiplicación de los mensajes & 11 \\
\hline Rapidez & 18 \\
\hline Facilita satisfacer demanda de información & 8 \\
\hline Medio masivo & 2 \\
\hline Inmediatez & 7 \\
\hline Actualidad & 6 \\
\hline Compartir & 17 \\
\hline $\begin{array}{l}\text { Dar a conocer su actividad social y } \\
\text { compartir información }\end{array}$ & 16 \\
\hline Experiencias & 3 \\
\hline Gratuidad & 17 \\
\hline Democratización & 13 \\
\hline Participación & 12 \\
\hline Apoyo social & 3 \\
\hline Democratización & 1 \\
\hline Transformador social & 1 \\
\hline Cooperar & 1 \\
\hline Segmentación & 12 \\
\hline Segmentación & 4 \\
\hline Conocer al público que le interesa nuestra labor & 6 \\
\hline Acercarnos al público más joven & 5 \\
\hline Imagen positiva, comprometida y responsable & 6 \\
\hline Nuevos formatos & 5 \\
\hline
\end{tabular}

Fuente: Elaboración propia
Tabla 2.Respuestas objetivadas del cuestionario sobre miedos e inconvenientes de las redes sociales

Concepto

Frecuencia

Falta de control 27

Uso incorrecto de datos e información 27

Imagen negativa de la ONG 2

Visto como un juego

Vulneración derechos 22

Pérdida del respeto (difamaciones) 10

Falta de intimidad 6

Protección de la infancia y de la mujer $\quad 18$

Banalización del trabajo 3

Violencia 2

\begin{tabular}{ll}
\hline Escasez de recursos & 2 \\
Pocos recursos humanos para trabajo & 13 \\
diario y continuo & 9 \\
Cuesta tiempo y esfuerzo & 7 \\
Nutrirla de información necesaria & 1 \\
\hline Desconfianza & 11 \\
Desconfianza & 5 \\
No saber a quién llega la información & 3 \\
Incertidumbre & 2 \\
Entrar en la conversación de una manera & 1 \\
transparente y sincera & 8 \\
\hline Saturación y Competencia & 5 \\
Dificultad de destacar y llamar la atención & 3 \\
Competencia & 2 \\
No ser pesados & 8 \\
\hline Inexperiencia & 1 \\
Desconocimiento de la propia herramienta & 6 \\
Falta de experiencia & 2 \\
\hline Segmentación & 5 \\
Por interés & 2 \\
Exclusiva participación con contacto previo & 1 \\
Por edad & 1 \\
\hline Otros diferente al receptor redes sociales & 1 \\
\hline Ninguno & 3 \\
\hline
\end{tabular}

Fuente: Elaboración propia 
Tabla 3. Presencia en redes sociales

\begin{tabular}{lll} 
Presencia en Redes Sociales & $\mathbf{N}^{\mathbf{0}}$ & $\%$ \\
\hline Sí & 20 & 57,14 \\
En qué redes & 49 & \\
Facebook & 17 & 85,00 \\
YouTube & 11 & 55,00 \\
Twitter & 7 & 35,00 \\
Tuenti & 6 & 30,00 \\
Flickr & 3 & 15,00 \\
Hi5 & 2 & 10,00 \\
MySpace & 1 & 5,00 \\
Linkedln & 1 & 5,00 \\
Menéame & 1 & 5,00 \\
\hline No & 12 & 34,28 \\
\hline No contesta & 3 & 8,57 \\
\hline TOTAL & 35 & 100,00 \\
\hline
\end{tabular}

Fuente: Elaboración propia

45\% de las ONG con presencia en redes indica estar solo en una, generalmente en Facebook por su grado de penetración y por cercanía con sus públicos objetivos; el 10\% manifiesta estar en dos; en tres, el $25 \%$ y en más de tres, el restante $20 \%$. Respecto a las expectativas que depositan las ONG en las acciones que desarrollan en las redes, la más destacada es la de interactuar con sus públicos en acciones de cooperación, participación y movilización (la señalan el 50\%), seguida de compartir informaciones, experiencias y conocimientos (25\%) y de un mayor alcance y presencia en medios (25\%).

En cuanto a la gestión de las redes (Tabla 4), el 75\% de las ONG que utilizan este medio indica que realizan actualizaciones de la información y lo hacen mayoritariamente en un intervalo de tiempo igual o menor a los dos o tres días. Esto pone de relieve una valoración significativa del poder de instantaneidad, tal y como ya pusieron de manifiesto en la valoración de las potencialidades del medio, cuando destacaron el valor de la inmediatez entre los tiempos de actualización y recuperación de la información. La tarea de actualización la realiza el responsable de comunicación, si bien un número considerable de encuestados no respondió a esta pregunta.
Tabla 4. Frecuencia y responsabilidad de actualización de la información

\begin{tabular}{lll}
$\begin{array}{l}\text { Actualización de la información } \\
\text { periódicamente }\end{array}$ & Frec. & $\%$ \\
\hline Sí & 15 & 75 \\
Diaria & 5 & 33,33 \\
Dos o tres días & 4 & 26,66 \\
Intenta hacerlo & 1 & 6,66 \\
No lo indica & 5 & 33,33 \\
\hline No & 4 & 20 \\
\hline No contesta & 1 & 5 \\
\hline TOTAL & 20 & 100 \\
\hline Responsabilidad de la & $\mathbf{N}{ }^{\circ}$ & $\%$ \\
actualización & & \\
\hline Responsable departamento & 10 & 50 \\
comunicación & & 10 \\
\hline Por un voluntario & 2 & 40 \\
\hline No contesta & 8 & 100 \\
\hline TOTAL & 20 & \\
\hline
\end{tabular}

Fuente: Elaboración propia

Por último, respecto a la medición de la eficacia de los mensajes en redes sociales, se constata que no es una actividad prioritaria de las organizaciones (Tabla 5). Las pocas que hacen un seguimiento (25\%) observan el número de visitas y, en menor medida, las personas registradas y los comentarios, lo que contrasta con el miedo referido anteriormente a perder el control de la comunicación. Se podría barajar la hipótesis de que es consecuencia de la ausencia de un auténtico profesional de redes sociales, un community manager.

\section{DE LA FASE CUALITATIVA}

Entre los resultados que se pueden extraer de los grupos de discusión destacan los diálogos mantenidos respecto a cuatro cuestiones: aspectos positivos de la comunicación a través de redes sociales; aspectos negativos de la misma; planificación de la comunicación en redes; y, por último, estrategia creativa en la comunicación a través de redes sociales. En los próximos párrafos lo ilustraremos con algunas de las opiniones expuestas por los participantes en los grupos de discusión y que son bastante coherentes con los resultados de la primera fase del estudio. 


\section{Tabla 5. Medición de la eficacia de los mensajes}

\begin{tabular}{lll}
$\begin{array}{l}\text { Medida de la incidencia de los } \\
\text { mensajes }\end{array}$ & Frec. & \% \\
\hline Sí & 5 & 25 \\
Número de visitas & 5 & 100 \\
Personas registradas & 3 & 60 \\
Comentarios & 3 & 60 \\
Informes periódicos (número & 2 & 40 \\
de seguidores, post y & & \\
respuestas) & 2 & 40 \\
Participación en eventos & 1 & 20 \\
Personas conectadas & 7 & 35 \\
\hline No & 8 & 40 \\
\hline No contesta & 20 & 100 \\
\hline TOTAL & & \\
\hline
\end{tabular}

Fuente: Elaboración propia

Aspectos positivos de la comunicación en redes sociales

Existen al menos tres importantes terrenos de consenso entre los gestores de la comunicación de las ONG. El primero se refiere a que las redes permiten una comunicación más participativa que otras herramientas, porque implican una comunicación más directa y transparente, lo que propicia el establecimiento de relaciones confiadas entre la organización y su público objetivo: "Lo que buscamos [... es hablar de tú a tú a las personas" (GD2, p. 7); "7 "hemos transmitido muchísimo hermetismo hasta hace poco. Es romper un muro. Es mostrar transparencia" (GD2, p. 21).

Valoran enormemente el hecho de tener un espacio propio en el que sensibilizar, informar y en el que los receptores puedan participar preguntando, proponiendo, opinando. En este sentido destacan que las redes sociales son útiles para comunicar hacia sus públicos, pero, sobre todo, para promover la comunicación participativa: "Es muy unidireccional la comunicación que hacemos. El objetivo de abrir estos nuevos canales [...] es un poco más de participación" (GD2, p. 12); "es salir de una situación de aislamiento porque tenemos una página web que no mira a los demás, sino que se mira el propio ombligo y no interacciona con la gente" (GD2, p. 9). Hay que tener en cuenta que estas organizaciones nacen desde la sociedad civil, de manera que perciben muy positivamente aquellos instrumentos de comunicación que permiten la implicación de capas amplias de la sociedad.
En segundo lugar, las ONG defienden que, gracias a esa comunicación participativa, las redes son útiles porque ayudan a promover el compromiso ciudadano con sus causas; en suma, para formar ciudadanos comprometidos: "Se está dando un uso lúdico a las redes sociales, pero luego hay otra serie de movimientos [...] que tienen sensibilidad" (GD2, p. 32); son una herramienta "para conseguir activistas online y offline [...] para que la gente se involucre: haga una donación o se haga socio" (GD2, p. 19). En consecuencia, creen en la utilidad de las redes para implicar a los ciudadanos en la mejora de la sociedad, haciéndolos partícipes de la actividad de la organización. Además, consideran que ese poder de las redes encuentra su explicación, principalmente, en su tremendo efecto multiplicador: por ejemplo, "en Facebook, detrás de cada persona hay otras 120 [...] Ése es el público al que puedes llegar" (GD1, p. 19); "cada persona transmite las cosas de la organización a sus amigos como si fueran suyas, por lo que tiene un peso importante" (GDl, p. 10).

En tercer lugar, a estas ventajas hay que añadir otras que tienen que ver con la diversidad de opiniones y con la posibilidad que tienen las ONG de abrir un debate social sobre las cuestiones en las que trabajan sus organizaciones. Así, no se trata solo del potencial de las redes para ayudar al desarrollo de las actividades propias de las ONG, sino también para incentivar el debate en la sociedad. El carácter dialógico de las redes se encuentra en la raíz de todo ello.

\section{Aspectos negativos de la comunicación en redes sociales}

En las conversaciones mantenidas en los grupos de discusión por parte de los miembros de los departamentos de comunicación de las ONG participantes, se detectaron también importantes temores respecto a las redes sociales. Entre los más recurrentes destacan, en primer lugar, los que tienen que ver con la pérdida de control de los procesos comunicativos en estos nuevos escenarios. Como escribe Baraybar, la comunicación es ahora diálogo y "al potenciar las relaciones se ha producido un nuevo ámbito en el que controlar la información por parte de la organización resulta imposible" (2009, p. 53). De ello son conscientes las ONG, que temen los comentarios negativos sobre sus miembros, sus proyectos, etc., especialmente aquellos contrarios a los valores o los fines de la organización.

A pesar de ello, hacen hincapié en que lo importante es canalizar y gestionar adecuadamente los comentarios negativos: "En todas las acciones que hemos hecho en los años que llevamos trabajando, solo hemos borrado los insultos" (GD2, p. 15); "le damos demasiada cancha al tema de los ataques, cuando los
7. Esta nomenclatura se utilizó para denominar de forma anónima a los participantes en los grupos de discusión. La primera parte se corresponde con el primer o el segundo grupo de discusión (GD1 o GD2 respectivamente) y la segunda con el número de página en el que ese texto consta en la transcripción de las conversaciones. 
que pueden tener mayores ataques [...] son las multinacionales" (GDl, p. 8). No defienden la censura o la negación de la realidad como estrategia para la gestión de los comentarios hostiles, sino más bien el aprovechamiento de los mismos para consolidar las relaciones entre la ONG y sus públicos.

En segundo lugar, otros aspectos negativos que perciben en el uso de las redes tienen más que ver con la falta de apoyo por parte de los estamentos directivos de sus organizaciones y la ausencia de definición estratégica de su uso. En cuanto a lo primero, persiste la consideración de las redes como un medio de menor impacto o relevancia que otros: "Más que el miedo nuestro [...] es el de la junta directiva, que además de considerarlo como un poco inútil y una pérdida de tiempo [...] lo ven hasta peligroso" (GD2, p. 14). En este sentido, no se valora la necesidad de incluir las redes en la estrategia comunicativa de la organización o ampliar el personal dedicado a las mismas: "Piensan que si tienen una persona que se dedica [a ello] es como si su sueldo se estuviera tirando a la basura" (GD1, p. 18).

En tercer lugar, las ONG señalan como un aspecto negativo un asunto puramente práctico, la multiplicidad de perfiles y la confusión que en torno a los mismos tienen algunas de estas ONG en las redes sociales: "Mucha gente se confunde pensando que es el perfil de nuestra organización pero de algún país de Sudamérica, a pesar de que el perfil indica [que pertenece] a España" (GD1, pp. 4-5); "el uso que se da [a las redes] supone algún problema porque llega a confundir a la gente" (GD1, p. 5). En muchas ONG han surgido perfiles específicos para determinados proyectos o secciones de la organización (voluntariado, juventud, etc.) y el resultado es que esa multiplicidad genera confusión en los públicos, dificultades para identificar cuál es el perfil real de la organización o dispersión de mensajes.

\section{Planificación de la comunicación en redes sociales}

Durante los grupos de discusión surgieron reflexiones en torno a la manera en que las ONG planifican estratégicamente su comunicación en las redes sociales y cómo deberían hacerlo. Para empezar, reconocen la necesidad de que la ONG diseñe un plan de comunicación online que permita establecer objetivos, centrar estrategias y permita, a posteriori, medir los resultados, algo que no existe en la mayoría de las ONG analizadas; como excepción están las más grandes, generalmente internacionales, y con departamentos de comunicación más estructurados Además, sería interesante que dentro de este plan de comunicación aparecieran referencias expresas a la gestión de la comunicación en redes, ya que es común escuchar en las ONG frases como: "Se está intentando promocionarlo, pero como tal, en el plan de comunicación tipificado, no [aparece reflejado]" (GD1, p 20). Hasta el momento, la comunicación que han realizado en las redes ha venido determinada generalmente por la intuición más que por la planificación. La estrategia dependerá de cada organización, de sus objetivos y forma de trabajar, pero quizás hay elementos comunes: "El camino no es recaudar, es interactuar para que luego la gente se sienta partícipe y que, igual que nos apoya en la red, nos apoye económicamente [...] Estamos sembrando, esa es la estrategia" (GD1, p. 19). "Si estás en la red, estás con todas las consecuencias. Si estás tienes que adaptarte a la red, no adaptar la red a ti" (GD1, p. 15)

La gran limitación es la escasez de recursos humanos y materiales a la que se enfrentan las ONG. No es una limitación nueva, que haya surgido con la aparición de las redes sociales; solo que ahora encuentran una misión más que deben cubrir y no encuentran cómo: "El tiempo que dedicamos es 'el que podemos" (GD1, p. 17); "creo que en eso [recursos necesarios] estamos todos en la misma situación, seamos más grandes o más pequeños" (GD2, p. 20).

Junto a estas limitaciones, durante las diluciones grupales aparece una especialmente interesante, porque será el caballo de batalla en el futuro: la medición de la eficacia. En principio se realiza de un modo cuantitativo, es decir, cuantificando el número de nuevos fans, de interacciones, etc. Existen herramientas que algunas ONG están utilizando ya "para trackear'el grado de conversiones desde ciberactivista a socio, del tráfico que llega a través de Facebook" (GD1, p 13), pero se trata de organizaciones muy excepcionales y reconocen que es algo "que tiene que desarrollarse mucho más" (GD1, p. 13).

\section{Estrategia creativa en la comunicación en redes sociales}

Finalmente, cabe detenerse en la estrategia creativa de la comunicación que desarrollan las ONG en redes sociales, ya que esta puede conllevar necesidades y características específicas cuando se difunde a través de estas plataformas. Entre las adaptaciones que han observado las organizaciones en su comunicación en redes está el que los mensajes deben ser más breves, directos y visuales que en otros medios; por esto, algunos de los representantes de las ONG hablaron de "banalización de la información": "En general el mensaje tiende a descafeinarse, sobre todo en temas que tienen cargas técnicas" (GD1, p. 14)

Al mismo tiempo, han observado que los mensajes deben ser más creativos y rompedores que los que elaboraban para otros medios: "Echode menos más agresividad" (GD1, p. 16); "el humor [...] sería muy positivo meterlo en la comunicación 
de las ONG, porque se nos ve siempre como un mundo un poco penoso" (GD1, p. 16). Los mensajes se deben adecuar al lenguaje de las redes y para eso quizás sea necesario romper con los esquemas de comunicación a los que las ONG están acostumbradas: "Me niego a considerar que la organización, por tener unos fines serios, está reservada a gente seria y actitudes serias" (GD2, p. 32).

\section{CONCLUSIONES Y DISCUSIÓN}

Se confirma que los usos que las ONG hacen de las redes sociales se relacionan con las percepciones que los responsables de las comunicaciones tienen de los aspectos positivos y negativos de las mismas, limitadas por la escasez de recursos humanos y materiales a la que se enfrentan y por la necesidad de incorporarlas en una planificación estratégica a mediano plazo, que permita ir avanzando en su uso sin descuidar otras formas de comunicación complementarias.

Las redes sociales son percibidas por las ONG como una herramienta idónea para desarrollar cauces de comunicación más participativos y directos que les permitan hablar de tú a tú con la sociedad civil, facilitando así un mayor compromiso ciudadano. Y, al mismo tiempo, ejercen un efecto multiplicador de sus mensajes, lo que en un primer momento servirá para invitar a los receptores a participar y a apoyar sus acciones comunicativas y, posteriormente, para desarrollar otras acciones de mayor compromiso, como donaciones, voluntariado, etc.

Pese a considerarse las redes un medio de gran relevancia, no se aprovechan todavía todas las oportunidades que ofrecen para la consecución de los objetivos de mejora y cambio social propios de las ONG. Esta insuficiente utilización de las redes conecta con el uso limitado que dichas ONG siguen haciendo de internet en general, como lo atestigua un reciente estudio de Montoliu i Riu (2012), cuyas conclusiones son coherentes con las aquí presentadas en el sentido de subrayar la infrautilización de la red para promover la interacción con la ciudadanía; cuestión que otros autores (Celaya, 2011) también señalan como dominantes en el uso que en general se hace de las redes sociales.

Existen, fundamentalmente, dos tipos de frenos internos que obstaculizan un uso más intensivo y eficaz de las redes en la comunicación de las ONG. Por una parte, la habitual falta de apoyo que muestran los estamentos directivos, que las siguen considerando un medio de menor visibilidad e impacto que otros más tradicionales, como la prensa o la televisión. Y, por otra, los insuficientes recursos con los que a menudo cuentan estas organizaciones para la gestión de su comunicación.
De igual manera, existen frenos externos a las propias organizaciones aunque intrínsecos a los medios sociales; por ejemplo, la dimensión interactiva y participativa de las redes, que los responsables de las ONG reconocen al mismo tiempo como la otra cara de la gran ventaja de estas plataformas. Esa dimensión interactiva la relacionan, sobre todo, con la pérdida de control de los procesos de comunicación que impulsan en las redes, a diferencia de lo que sucede en otros medios.

La existencia de multiplicidad de perfiles de la misma organización en la misma red o diferentes redes sociales, por razón de existencia de múltiples organizaciones locales o internacionales, genera confusión en los públicos y esto preocupa a los gestores de la comunicación.

La incorporación de las redes sociales a la planificación estratégica de la comunicación de las ONG es fundamental porque, hasta el momento y salvo excepciones, se han movido de manera intuitiva, funcionando con esquemas de ensayo y error. Si no se definen estrategias y objetivos previos, no se podrá aprovechar todas las potencialidades de las redes.

Asimismo, la medición de la eficacia de los mensajes en redes sociales no es una actividad prioritaria de las ONG; prueba de ello son las pocas organizaciones que lo hacen y la escasez de sus observaciones, que apenas pasan de medir el número de fans o visitas que reciben en el perfil. El futuro ha de pasar por el diseño y perfeccionamiento en los sistemas de medición de eficacia, para que las experiencias puestas en marcha por el tercer sector a través de las redes puedan ser evaluadas tanto cuantitativa como cualitativamente.

Por último, se ha observado la urgencia de adaptar en mayor grado los mensajes al lenguaje propio de las redes sociales, así como la importancia de la creatividad y la innovación en los contenidos parallegar de manera más eficaz a los públicos. Creatividad basada en nuevos diseños llamativos, cercanos y modernos; nuevos formatos breves y visuales; contactos más directos con públicos más específicos. Se trata de una conclusión corroborada también por otros estudios, de ahí que en ocasiones se hable de una "comunicación previsible donde las ONG cuentan lo que hacen, lo que hace falta hacer, pero no construyen mensajes que capten la atención y circulen por la red" (Arroyo, Baños y Rodríguez, 2012, p. 15).

A pesar de los datos obtenidos en esta investigación y su coherencia con otros estudios recientes hechos en otros países (Waters, Burnett, Lamm y Lucas, 2009; Quinn y Berry, 2010), los cambios se suceden de forma muy acelerada en el mundo de las redes sociales, por lo que son necesarias periódicas réplicas de la investigación o trabajos complementarios a la misma, 
para así permitir una actualización constante de los resultados obtenidos. De igual modo, las ONG se están viendo forzadas a adaptarse a este cambio constante, jugando en ocasiones en desventaja porque, en términos generales, llevan un importante retraso en el uso de las redes sociales con respecto a otras organizaciones de nuestra sociedad.

En este sentido será clave cómo las ONG adapten sus usos y presupuestos de comunicación a la nueva realidad derivada de la crisis económica que se lleva agudizando en España desde el momento de la realización del estudio de campo del trabajo aquí recogido. Las dificultades presupuestarias de estas organizaciones, influidas entre otras razones por la caída de los fondos públicos destinados a estos fines, influirá decisivamente en cómo planifiquen su comunica-

\section{REFERENCIAS}

Aguadero, F. (1993). Comunicación social integrada: un reto para la organización. Barcelona: Consejo Superior de Comunicación y Relaciones Públicas en España.

Angulo, G. (1998) El apoyo público a la cooperación al desarrollo. Revista Española de desarrollo y cooperación, 2, 127-144

Aranguren Gonzalo, L. (1998). Reinventar la solidaridad: voluntariado y educación. Madrid: PPC.

Arroyo, I., Baños, M. y Van-Wyk, C. (2013) Análisis de los mensajes audiovisuales del Tercer Sector enYouTube. Revista Latina de Comunicación Social, 68, 328-354. Recuperado el 25 de mayo de 2013, de http://www.revistalatinacs.org/068/paper/980_ Madrid/14_Arroyo.html DOI: 10.4185/RLCS2013-980/CrossRef link

Arroyo, I., Baños, M. \& Rodríguez, T. C.. (2012). Modelo de investigación de las piezas audiovisuales de las organizaciones del tercer sector en redes sociales. En M. Moragas, B. López, \& R. García, Comunicación y Riesgo. Tarragona: Actas del Congreso AE-IC 2012 AE-IC, 1-18 ción y en el papel cada vez más importante que podrían adquirir los nuevos medios, en especial por la razón señalada al principio de este artículo: los reducidos costos que supone la implementación de acciones de comunicación en comparación con los que se necesitan para llevar a cabo otras acciones convencionales en medios más tradicionales. Ahora bien, tampoco conviene olvidar que, aunque estos nuevos medios supongan costos mucho más reducidos, no cabe perder de vista que muchas ONG están pasando por difíciles situaciones económicas que les están obligando a reducir sus estructuras, plantillas, proyectos, etc., por lo que será difícil que la comunicación en redes sociales pueda no verse afectada por esta situación, pudiéndose reducir más los recursos destinados a esos nuevos medios.

Arroyo, I \& Martín Nieto, R. (2011). La utilización de Internet en la comunicación expresiva de las ONG: estudio exploratorio comparativo entre Argentina y España. Zer, 16, 243-263.

Asociación para la Investigación de Medios de Comunicación, Navegantes en la Red. (2010). $13^{a}$ encuesta AIMC a usuarios de Internet, recuperado de: http://download.aimc.es/aimc/ navred2010/macro2010.pdf, fecha consulta: julio 2011

Baraybar, A. (2009). Conectando valores. Las nuevas estrategias de la comunicación online en el Tercer Sector, Icono 14, 13, 67-55

Bericat, E. (1998). La integración de los métodos cuantiativo y cualitativo en la investigación social, Barcelona: Ariel.

Berrios, O. (2005). El papel de los blogs en la acción social. Blogs en ONG, una oportunidad poco conocida. Telos, 65, s. p. Recuperado de:http://sociedadinformacion.fundacion. telefonica.com/telos/articulocuaderno.asp@ idarticulo $=6 \&$ rev=65.htm. Fecha de consulta: 1 de febrero de 2013. 
Celaya, J. (Año). Cultura digital en redes sociales. Escasa creación original, colaborativa, participativa, Telos, 88. Recuperado de: http:// sociedadinformacion.fundacion.telefonica. com/DYC/TELOS/ResultadoBsquedaTELOS/ DetalleArticuloTelos_88TELOS_ DOSSIERPV3/seccion $=1227$ \&idioma $=$ es $_{\text {_ }}$ ES\&id $=2011072809100001 \&$ activo $=6$. do. Fecha consulta: 1 de diciembre de 2011.

Cerdá Morales, S. (2003). Las ONGD como agentes de la cooperación y la ayuda humanitaria. El caso español. Barcelona: TDX. Recuperado de http:// www.tesisenred.net/handle/10803/4117

Citizen Schools \& Public Learning Media Lab. (2008). Resultados de la encuesta sobre el uso de la web social en las organizaciones. Recuperado de: http://yankana. org/index.php/2008/04/resultados-de-la-encuestasobre-el-uso-de-la-web-social-en-organizaciones/, fecha de consulta: 1 de noviembre de 2011.

Erro, J. (2002).Comunicación, Desarrollo y ONGD. Bilbao: Hegoa.

Ferreras Rodríguez, E. M. (2011) Redes sociales y cambio social. El movimiento 15-M y su evolución en Twitter. Telos, 89, s.p. Recuperado de: http://sociedadinformacion.fundacion. telefonica.com/seccion $=1266 \&$ \&idioma $=$ es_ ES\&id $=2011102410330001 \&$ activo $=6$. do. Fecha de consulta: 8 de enero de 2012 .

Gaitán Moya, J. Y Piñuel Raigada, J. (1998). Técnicas de investigación en comunicación social. Elaboración y registro de datos. Madrid: Síntesis.

Grzybowski, C. (2001). Las organizaciones no gubernamentales y la comunicación de masas: posibilidades de movilización. Comunicar, 16, 25-32.

Herranz de la Casa, J. M, y Cabezuelo Lorenzo, F. (2009). Comunicación y transparencia en las organizaciones sociales. Los blogs como generadores de transparencia en las ONG. Icono $14,13,172-194$.
Herranz de la Casa, J. M (2006). La gestión de la comunicación como elemento generador de transparencia en las organizaciones no lucrativas [en línea]. XI Jornadas de Investigadores en Economía Social y cooperativa, CIRIEC, Santiago de Compostela 25-27de octubre.

Laporte, J. M. (2001). Principios de comunicación interna en el tercer sector. Comunicación y Sociedad, XIV, 1, 7-27.

Marí Sáez, V. (2001). Contra la evaporación de la dimensión política de la comunicación. Movimientos sociales, ONG y usos de Internet. Zer, 22, 453-471

Martín Nieto, R. (2010). La comunicación web del tercer sector: análisis de los sitios web de las ONGD calificadas por la AECID (tesis doctoral). Madrid: Universidad Rey Juan Carlos.

Montoiu I Riu, L. (2012). Las ONG en la Red. Análisis de la presencia, la interactividad y la participación en Internet de las entidades del tercer sector catalán. En M. Moragas, B. López, \& R. García, Comunicación y Riesgo. Tarragona: Actas del Congreso AE-IC, AE-IC, 1-12.

Quinn, L. y Berry, A. (2010). The nonprofit social media decision guide. Portland: Idealware.

Ramil, X. (2005). Las ONG comienzan a fortalecer sus redes sociales mediante las nuevas tecnologías. Canalsolidario.org. Recuperado de: http://www. canalsolidario.org/noticia/las-ong-comienzan-afortalecer-sus-redes-sociales-mediante-las-nuevastecnologias/6273. Consulta: mayo 2008.

Sabre, M. E. (2011). Implicación y encuadre como factores de eficacia en los mensajes publicitarios de las ONG. Comunicación y Sociedad, XXIV, 1, 269-301.

Sampedro, V. (2006). Comunicación y tercer sector ¿Redes de nudos o vacíos? Nuevas tecnologías y tejido social. Documentación Social, 140, s.p. Recuperado de: http://www.nodo50.org/ multitudesonline/Sampedro_DEFINITIVO.pdf-. Consulta: 10 de enero de 2012. 
Soler, P. (1999). La investigación cualitativa en marketing y publicidad. El grupo de discusión y el análisis de datos. Barcelona: Paidós

Valles, M. (2000). Técnicas cualitativas de investigación social. Reflexión metodológica y práctica profesional. Madrid: Síntesis.

Waters, R. D., Burnett, E., Lamm A. y Lucas, J. (2009). Engaging stakeholders through social networking: How nonprofit organizations are using Facebook. Public Relations Review, 35, 102-106.
Zafra, R. (2010). Un cuarto propio conectado. (Ciber) espacio y (auto)gestión del yo. Madrid: Fórcola.

Zorn, T. E., Flanagin, A. J. y Shoham, M. (2011). Institutional and no institutional influences on information and communication technology adoption and use among non-profit organizations Human Communication Research, 37, 1-33.

\section{SOBRE LOS AUTORES:}

Isidoro Arroyo Almaraz es doctor en Ciencias de la Información por la U.C.M.; máster en Técnicas de Comunicación en Servicios Sociales UCM-IMSERSO / Profesor Titular Universidad Rey Juan Carlos; Profesor Visitante en diversas universidades. / Investigador invitado en la Universidad Nacional de Córdoba (República de Argentina).

Antonio J. Baladrón Pazos es doctor en Publicidad, Universidad Rey Juan Carlos, licenciado en Periodismo y RR.PP. / Profesor Universidad Rey Juan Carlos de Madrid, España. / Autor de los libros “Consumo y publicidad para inmigrantes. Claves para dirigirse a un mercado emergente" y “Violencia y publicidad televisiva. De la violencia como recurso creativo a la publicidad como violencia".

Rebeca Martín Nieto es doctora en Ciencias de la Comunicación y licenciada en Comunicación Audiovisual. / Profesora Visitante en la Universidad Rey Juan Carlos. /Áreas de investigación: comunicación social, persuasiva, redes sociales. 